\title{
MIXING OF HEAVY BARYONS IN THE BAG MODEL CALCULATIONS
}

\author{
A. Bernotas and V. Šimonis \\ Vilnius University Institute of Theoretical Physics and Astronomy, A. Goštauto 12, LT-01108 Vilnius, Lithuania \\ E-mail: bernotas@itpa.lt, simonis@itpa.lt
}

Received 4 March 2008; accepted 9 June 2008

\begin{abstract}
Spin-spin interaction causes the mixing between ground state wave functions of baryons containing three quarks of different flavours. We examine the effect of this mixing on the baryon masses in the framework of the modified bag model.
\end{abstract}

Keywords: bag model, heavy quarks, heavy baryons, wave function mixing

PACS: $12.39 . \mathrm{Ba}, 14.20 . \mathrm{Lq}$, 14.20.Mr

\section{Introduction}

Invented more than 30 years ago, the MIT bag model [1-3] still serves as an useful method which yields reasonable predictions for a variety of hadronic properties (at least for the ground states). Various aspects of the bag model are discussed in a number of review papers [4-6]. Originally the model was designed for the ultrarelativistic case of the light quarks and was rather successful in describing the low-lying hadron spectrum. Although the first straightforward application of the model to calculate the spectrum of the hadrons containing heavy quarks was of very limited success because of evident disagreement between calculated and observed data, later on the bag model was adjusted to incorporate the heavy quarks. It was recognized that the reconciliation between the bag model and the heavy quark physics could be achieved by taking into account the socalled c. m. m. (centre of mass motion) correction. For the hadrons containing only one heavy quark there exists an approximate solution of the c. m. m. problem one can simply associate the centre of mass with the heavy quark and fix it $[7,8]$. For the baryons containing two heavy quarks one can proceed in a similar way assuming the heavy quarks to form the doubly-heavy diquark and then put this object at the centre of the bag [9]. The advantage of such approach is the simple and clear physical picture, but the price for this simplicity is three more ore less related bag models: one for the hadrons consisting of light quarks (in this case the role of c. m.m. correction is partly played by the so-called zero-point energy), one for hadrons containing single heavy quark, and one for the baryons containing two heavy quarks. Moreover, there remains the case of the baryons consisting of three heavy quarks which needs special treatment. Another rather popular method to deal with c. m. m. problem is to employ a wave-packet ansatz [10]. Both approaches give only approximate solutions to the problem. However, the second seems to be more universal and could be preferable in the case one tries to obtain the unified description of the light and heavy hadrons. The c. m.m. corrections is not all the story, and in order to have plausible unified description of light and heavy hadrons (mesons and baryons) in the framework of the bag model some other QCD inspired improvements such as running coupling constant and running quark masses are necessary [11].

When the number of quark flavours increases we are confronted with additional problem which needs some clarification. For the spin- $1 / 2$ baryons containing three quarks of different flavours there exist two states with the same spin and parity. We can construct the set of orthogonal wave functions by assuming the first two quarks to be in the relative spin- 0 or spin- 1 state, respectively. In general case the physical states would be the linear combinations of these mathematical states

$$
\begin{gathered}
|B\rangle=C_{1}\left|\left(q_{1} q_{2}\right)^{0} q_{3}\right\rangle+C_{2}\left|\left(q_{1} q_{2}\right)^{1} q_{3}\right\rangle, \\
|B\rangle^{\prime}=C_{2}\left|\left(q_{1} q_{2}\right)^{0} q_{3}\right\rangle-C_{1}\left|\left(q_{1} q_{2}\right)^{1} q_{3}\right\rangle .
\end{gathered}
$$

The mixing mechanism depends on the model and approximation used. In the MIT bag it would be the 
hyperfine colour-magnetic interaction. The same is true in the ordinary constituent quark model as well as in almost all variants of the potential model. Some estimate of the state mixing is also possible in the heavy quark effective theory [12].

Because of the ambiguity in how the quarks are to be ordered in the mathematical wave functions $\left|\left(q_{1} q_{2}\right)^{S} q_{3}\right\rangle$ the expansion in Eqs. (1) is not unique. If one does not want to bother about the quark ordering, one can simply diagonalize the 2 nd order interaction energy matrix [13]. Since such a procedure has not become a common practice, the very natural question arises, how some authors have managed to avoid this state mixing problem. The answer was given a long time ago in the Ref. [14]. The authors of that paper have shown that for the interaction energies with quark mass dependence $\sim 1 /\left(m_{a} m_{b}\right)$ an optimal quark ordering scheme could be found. The prescription is to pick the closest in mass quarks as the first two in the wave function $\left|\left(q_{1} q_{2}\right)^{S} q_{3}\right\rangle$. Then the mixing of the states with different $S$ values is small, and the effect of this mixing on baryon masses is negligible. Strictly speaking, such mixing exists even in the light baryon sector between the wave functions from which the physical states $\Sigma^{0}$ and $\Lambda^{0}$ are constructed. Because of the approximate isotopic symmetry this mixing is small, and if we are interested only in the calculation of baryon masses, we can safely ignore this effect. The explicit calculations with the isospin-symmetry violating terms taken into account show that the mixing is indeed small [15], as expected. In the sector of charmed baryons there are also rather strong indications that the mixing between $\Xi_{c}$ and $\Xi_{c}^{\prime}$ baryons is small, with negligible shifts in the masses of these hadrons again [16].

And what could be said about the wave function mixing in the framework of the bag model? Of course, we expect that all reliable models of hadron structure yield similar results. However, we cannot apply the results of Ref. [14] directly because the dependence of the interaction energy on quark masses in the bag model is somewhat more complicated. For example, the values of light quark masses in the bag model could be set to zero, while in the nonrelativistic models these values approach one-third of the nucleon mass. A simple way to make the things clear is to perform direct calculations in the bag model taking the mixing interaction into account. This means that in the calculations of baryon energy the off-diagonal matrix elements of the colourmagnetic interaction should be included.

In this paper we are going to examine the mixing of the ground state baryon wave functions in the frame- work of the modified MIT bag model. In the next section we give a short description of the model we are dealing with. The concluding section contains the results of our investigation accompanied by the discussion and some additional remarks on the validity of approaches with and without mixing.

\section{The model}

The ground state energy of the hadron defined in the static spherical cavity approximation is given by

$$
E=\frac{4 \pi}{3} B R^{3}+\sum_{i} n_{i} \varepsilon_{i}+\Delta E,
$$

where $B$ is the bag constant, $R$ is the bag radius, $\varepsilon_{i}$ is eigenenergy of the $i$ th quark in the cavity, and $\Delta E$ stands for the interaction energy. $\Delta E$ consists of colour-electric and colour-magnetic parts as described, for example, in Ref. [11] in detail. For our purpose the most important is the contribution of the colourmagnetic interaction, which in the case of the baryons containing three distinct quarks can be written as

$$
\Delta E_{m}=\alpha_{\mathrm{c}}(R) \sum_{j>i} a_{i j} M_{i j}\left(m_{i}, m_{j}, R\right) .
$$

Here $\alpha_{\mathrm{c}}(R)$ is the running strong coupling constant. The function $M_{i j}\left(m_{i}, m_{j}, R\right)$ depends on quark masses and hadron bag radius and it can be calculated explicitly. Parameters $a_{i j}$ specify the spin dependence of the interaction energy between quarks $q_{i}$ and $q_{j}$. They are proportional to the matrix elements $\left\langle\left(q_{1} q_{2}\right)^{S_{1}} q_{3}\right|\left(\sigma_{i}\right.$. $\left.\sigma_{j}\right)\left|\left(q_{1} q_{2}\right)^{S_{2}} q_{3}\right\rangle$, where $\sigma_{i}$ are appropriate spin generators. These coefficients can be calculated straightforwardly using algebraic technique, as described in the Ref. [17], and the transformation of the basis [18]

$$
\begin{aligned}
\left|\left(q_{1} q_{2}\right)^{J_{12}} q_{3}\right\rangle^{J}= & \sum_{J_{13}}(-1)^{j_{2}+j_{3}+J_{12}+J_{13}} \\
& \times \sqrt{\left(2 J_{12}+1\right)\left(2 J_{13}+1\right)} \\
& \times\left\{\begin{array}{ccc}
j_{2} & j_{1} & J_{12} \\
j_{3} & J & J_{13}
\end{array}\right\}\left|\left(q_{1} q_{3}\right)^{J_{13}} q_{2}\right\rangle^{J},
\end{aligned}
$$

where necessary. The results are presented in Table 1 , where for simplicity the abbreviations $\left|J_{12}\right\rangle=$ $\left|\left(q_{1} q_{2}\right)^{J_{12}} q_{3}\right\rangle$ are used.

The relation between the calculated bag-model energy $E$ and the hadron mass $M$ is given by

$$
E=\int \mathrm{d}^{3} s \Phi_{P}^{2}(s) \sqrt{M^{2}+s^{2}},
$$


Table 1. Parameters which specify the colour-magnetic interaction energy of baryons consisting of three distinct quarks.

\begin{tabular}{cccc}
\hline & $a_{12}$ & $a_{13}$ & $a_{23}$ \\
\hline$\left\langle 0\left|a_{i j}\right| 0\right\rangle$ & -3 & & \\
$\left\langle 1\left|a_{i j}\right| 1\right\rangle$ & 1 & -2 & -2 \\
$\left\langle 0\left|a_{i j}\right| 1\right\rangle$ & & $\sqrt{3}$ & $-\sqrt{3}$ \\
\hline
\end{tabular}

where $\Phi_{P}(s)$ is a Gauss profile

$$
\Phi_{P}(s)=\left(\frac{3}{2 \pi P^{2}}\right)^{3 / 4} \exp \left(-\frac{3 s^{2}}{4 P^{2}}\right) .
$$

The effective momentum $P$ specifies the momentum distribution and is defined as

$$
P^{2}=\gamma \sum_{i} n_{i} p_{i}^{2}
$$

Here $p_{i}$ are the momenta of the quarks. The c. m. m. parameter $\gamma$ is to be determined in the fitting procedure. For the baryons containing $b$-quarks the relation (5) gives practically the same results as the familiar Einstein relation [19]

$$
M^{2}=E^{2}-P^{2} .
$$

In the presence of $b$-quarks we prefer to use this simple relation instead of rather cumbersome procedure based on Eq. (5). For the running coupling constant $\alpha_{\mathrm{c}}(R)$ and running quark mass $\bar{m}_{f}(R)$ we use the following expressions:

$$
\begin{gathered}
\alpha_{\mathrm{c}}(R)=\frac{2 \pi}{9 \ln \left(A+R_{0} / R\right)}, \\
\bar{m}_{f}(R)=\tilde{m}_{f}+\alpha_{\mathrm{c}}(R) \cdot \delta_{f},
\end{gathered}
$$

where $R_{0}$ is the scale parameter analogous to QCD constant $\Lambda$. Parameter $A$ helps us to avoid divergences when $R \rightarrow R_{0}$. For each quark flavour we have two free parameters $\widetilde{m}_{f}$ and $\delta_{f}$ to be adjusted.

Let us summarize our zoo of model parameters. These are the bag constant $B$, the c.m.m. parameter $\gamma$ which determines the strength of the c. m.m. corrections, two parameters $\left(A\right.$ and $R_{0}$ ) from the running coupling constant parametrization, and finally six parameters $\left(\widetilde{m}_{s}, \delta_{s}, \widetilde{m}_{c}, \delta_{c}, \widetilde{m}_{b}, \delta_{b}\right)$ necessary to define the running quark mass functions $\bar{m}_{f}(R)$. The light (up and down) quarks are taken to be massless. To fix the parameters $B, \gamma, A$, and $R_{0}$ the experimentally observed masses of the light hadrons $(N, \Delta, \pi$, and the average mass of the $\omega-\rho$ system) were chosen. To fix the mass function parameters $\widetilde{m}_{f}, \delta_{f}$ for each quark flavour we have employed the masses of corresponding lightest vector mesons $(\phi, J / \psi, \Upsilon)$ and the mass values of the lightest baryons $\Lambda_{f}$ containing the quark $q_{f}$ of the corresponding flavour. We employ the same fitting procedure as in our previous work [11], and the values of the parameters to be used as the input in the bag model calculations are: $B=7.597 \cdot 10^{-4} \mathrm{GeV}^{4}$, $R_{0}=2.543 \mathrm{GeV}^{-1}, A=1.070, \gamma=1.958, \widetilde{m}_{s}=$ $0.161 \mathrm{GeV}, \delta_{s}=0.156 \mathrm{GeV}, \widetilde{m}_{c}=1.458 \mathrm{GeV}$, $\delta_{c}=0.112 \mathrm{GeV}, \widetilde{m}_{b}=4.793 \mathrm{GeV}, \delta_{b}=0.061 \mathrm{GeV}$. The parameters $B, R_{0}, A, \gamma, \widetilde{m}_{s}, \delta_{s}$ are the same as in Ref. [11]. The numerical values of the remaining four parameters $\left(\widetilde{m}_{c}, \delta_{c}, \widetilde{m}_{b}, \delta_{b}\right)$ differ slightly from the corresponding values presented in [11] because in the present work we have used new more accurate values of $\Lambda_{c}(2.286 \mathrm{GeV})$ [20] and $\Lambda_{b}(5.620 \mathrm{GeV})$ [21] masses.

\section{Results and discussion}

Let us proceed to the discussion of our main point of concern - the wave function mixing of heavy baryons in the bag model calculations. The ground state baryons we are interested in are $\Xi_{c}, \Xi_{c}^{\prime}, \Xi_{c}^{*} ; \Xi_{b}, \Xi_{b}^{\prime}, \Xi_{b}^{*} ; \Xi_{b c}, \Xi_{b c}^{\prime}$, $\Xi_{b c}^{*}$; and $\Omega_{b c}, \Omega_{b c}^{\prime}, \Omega_{b c}^{*}$. The mixing is possible only between the spin- $1 / 2$ states $\left(\Xi_{c}\right.$ and $\Xi_{c}^{\prime}$, for example). In order to calculate the masses of all these baryons we use the bag model parameters listed at the end of the preceding section. For the spin-3/2 states denoted as $|\ldots\rangle^{*}$ the calculation procedure is exactly the same as adopted in the paper [11]. We minimize the energy $E_{B^{*}}$ of each such baryon as a function of the bag radius $R$ and then apply Eq. (5) (for $\Xi_{c}^{*}$ ) or Eq. (8) (for $\Xi_{b}^{*}, \Xi_{b c}^{*}$, and $\Omega_{b c}^{*}$ ) to determine the corresponding baryon masses. For the spin- $1 / 2$ states $|\ldots\rangle$ and $|\ldots\rangle^{\prime}$ the procedure differs only in the choice of the energy function to be minimized. In this case we use the trace of the energy matrix $E_{B}+E_{B^{\prime}}$ which remains invariant under state mixing. Then we calculate the diagonal and off-diagonal matrix elements of the interaction energy, diagonalize the energy matrix, and use Eq. (5) or (8) again to determine the masses of the physical baryons. To gain some insight how the things look like we present some intermediate results of calculations in the Tables 2, 3. In the first two rows of these tables we give the c. m. m. uncorrected energy values $E(1)$ and $E(0)$ corresponding to the mathematical wave functions in which the first two quarks in the spin coupling scheme $\left(q_{1} q_{2}\right)^{S} q_{3}$ are in the spin- 1 and spin- 0 states. The last two rows contain the squared wave function expansion coefficients obtained after matrix diagonalization. The symbols $b, c, s$ denote the bottom, charmed, and strange quarks, respectively, and for the sake of simplicity the symbol $u$ is used for both light (up or down) quarks. 
Table 2. Dependence of the c. m. m. uncorrected energies (in $\mathrm{GeV}$ ) before the matrix diagonalization on the arangement of quarks for the $\Xi_{c}, \Xi_{c}^{\prime}$ (columns 2-4) and $\Xi_{b}, \Xi_{b}^{\prime}$ (columns 5-7) baryons. The last two rows contain the squared expansion coefficients $C_{1}^{2}, C_{2}^{2}$ of the wave functions obtained after matrix diagonalization.

\begin{tabular}{lllllll}
\hline$\left(q_{1} q_{2}\right) q_{3}$ & $(u s) c$ & $(u c) s$ & $(s c) u$ & $(u s) b$ & $(u b) s$ & $(s b) u$ \\
\hline$E(1)$ & 2.886 & 2.840 & 2.831 & 6.086 & 6.013 & 6.010 \\
$E(0)$ & 2.818 & 2.865 & 2.874 & 5.987 & 6.059 & 6.062 \\
$C_{1}^{2}$ & 0.9950 & 0.3139 & 0.1912 & 0.9997 & 0.2656 & 0.2347 \\
$C_{2}^{2}$ & 0.0050 & 0.6861 & 0.8088 & 0.0003 & 0.7344 & 0.7653 \\
\hline
\end{tabular}

Table 3. Dependence of the c. m. m. uncorrected energies (in GeV) before the matrix diagonalization on the arangement of quarks for the $\Xi_{b c}, \Xi_{b c}^{\prime}$ (columns 2-4) and $\Omega_{b c}, \Omega_{b c}^{\prime}$ (columns 5-7) baryons. The last two rows contain the squared expansion coefficients $C_{1}^{2}$, $C_{2}^{2}$ of the wave functions obtained after matrix diagonalization.

\begin{tabular}{lllllll}
\hline$\left(q_{1} q_{2}\right) q_{3}$ & $(u c) b$ & $(u b) c$ & $(c b) u$ & $(s c) b$ & $(s b) c$ & $(c b) s$ \\
\hline$E(1)$ & 7.078 & 7.050 & 7.041 & 7.247 & 7.224 & 7.217 \\
$E(0)$ & 7.035 & 7.062 & 7.072 & 7.212 & 7.235 & 7.241 \\
$C_{1}^{2}$ & 0.9833 & 0.3693 & 0.1474 & 0.9872 & 0.3538 & 0.1591 \\
$C_{2}^{2}$ & 0.0167 & 0.6307 & 0.8526 & 0.0128 & 0.6462 & 0.8409 \\
\hline
\end{tabular}

The inspection of results in Tables 2 and 3 shows a striking dependence of the calculated energies on the quark ordering. As one can see, the wave function with two first quarks in the relative spin-0 state has the lowermost energy only when the heaviest quark (e.g., $b$-quark) is picked up as the third in the corresponding spin coupling scheme $\left(q_{1} q_{2}\right)^{S} q_{3}$. This is the only case when the traditional prescription

$$
|\ldots\rangle=\left|\left(q_{1} q_{2}\right)^{0} q_{3}\right\rangle, \quad|\ldots\rangle^{\prime}=\left|\left(q_{1} q_{2}\right)^{1} q_{3}\right\rangle
$$

could be maintained, because the energy matrix diagonalization leads to negligible changes of the initial energy values. The direct calculations show that even in the most problematic case of the $\Xi_{b c}-\Xi_{b c}^{\prime}$ system the difference between energy values before and after diagonalization does not exceed $1 \mathrm{MeV}$ and is obviously much smaller than the systematic uncertainties of the model. So, if one is interested only in the baryon mass spectra, one can adopt the prescription (11), construct the optimal basis by arranging the quarks in increasing order of their masses, and never bother about the diagonalization of the energy matrix anymore. At this point a remark is necessary. One must be very cautious when dealing with other baryon parameters (such as magnetic moments, for example). As it was shown in Ref. [14], the wave function mixing may change the values of the calculated magnetic moments substantially even when the optimal basis is used. Although this problem is beyond the scope of the present paper, it is worth attention, and we are going to return to this question in the future.
Table 4. Masses of $\Xi_{c}, \Xi_{c}^{\prime}, \Xi_{c}^{*}$ and $\Xi_{b}, \Xi_{b}^{\prime}, \Xi_{b}^{*}$ baryons (in GeV). The row denoted as Bag contains the results obtained in our work. The row Exp contains averaged over the isodoublet experimental energy values.

\begin{tabular}{lllllll}
\hline Particle & $\Xi_{c}$ & $\Xi_{c}^{\prime}$ & $\Xi_{c}^{*}$ & $\Xi_{b}$ & $\Xi_{b}^{\prime}$ & $\Xi_{b}^{*}$ \\
\hline Bag & 2.468 & 2.546 & 2.638 & 5.809 & 5.911 & 5.944 \\
{$[23]$} & 2.481 & 2.578 & 2.654 & 5.812 & 5.937 & 5.963 \\
{$[25]$} & 2.474 & 2.578 & 2.655 & 5.808 & 5.946 & 5.975 \\
{$[26,27]$} & 2.468 & 2.582 & 2.651 & 5.810 & 5.955 & 5.984 \\
Exp & 2.469 & 2.577 & 2.646 & - & - & - \\
\hline
\end{tabular}

Table 5. Masses of $\Xi_{b c}, \Xi_{b c}^{\prime}, \Xi_{b c}^{*}$ and $\Omega_{b c}, \Omega_{b c}^{\prime}, \Omega_{b c}^{*}$ baryons (in $\mathrm{GeV}$ ). The row denoted as Bag contains the results obtained in our work.

\begin{tabular}{lllllll}
\hline Particle & $\Xi_{b c}$ & $\Xi_{b c}^{\prime}$ & $\Xi_{b c}^{*}$ & $\Omega_{b c}$ & $\Omega_{b c}^{\prime}$ & $\Omega_{b c}^{*}$ \\
\hline Bag & 6.846 & 6.891 & 6.919 & 6.999 & 7.036 & 7.063 \\
{$[22]$} & 6.82 & 6.85 & 6.90 & 6.93 & 6.97 & 7.00 \\
{$[24]$} & 6.933 & 6.963 & 6.980 & 7.088 & 7.116 & 7.130 \\
{$[26]$} & 7.029 & 7.053 & 7.083 & 7.126 & 7.148 & 7.165 \\
{$[9]$} & 6.838 & 7.028 & 6.989 & 6.941 & 7.116 & 7.077 \\
\hline
\end{tabular}

Before going to the concluding remarks we want to compare the masses of baryons calculated in our work with the results obtained in other models and experimental data where available. We have chosen for the sake of comparison the baryon mass estimates in nonrelativistic [22] and relativistic [23,24] potential models obtained in the quark-diquark approximation, the estimates obtained in the quark-diquark approximation of the bag model [9], the calculations in the simplified variational approach [25], and predictions provided using various sum rules based partially on the heavy quark symmetry considerations $[26,27]$. The experimental values are taken from the Particle Data Tables [28]. The data for the baryons of $\Xi_{Q}$ type are presented in Table 4 and for the $\Xi_{Q_{1} Q_{2}}, \Omega_{Q_{1} Q_{2}}$ type baryons in Table 5 .

From Table 4 it is seen that for the baryons containing one heavy quark all approaches give rather similar qualitative picture. Inspection of the $\Xi_{Q}-\Xi_{Q}^{*}$ hyperfine mass splitting indicates that in our version of the bag model the interaction energies for these baryons could be slightly underestimated. Comparison with experiment also shows that all approaches give reasonable results. One could even insist that owing to the approximate nature of the models the agreement with experiment (though not excellent) is surprisingly good. Such success gives us some confidence that we are on the right path in understanding the properties of heavy baryons.

For the baryons with two heavy quarks the situation is somewhat different. As seen from Table 5, all but one approaches give similar qualitative pictures of the 
baryon spectra again. A striking exception is the results obtained in the paper [9] (the reversed order of the $\Xi_{b c}^{\prime}, \Xi_{b c}^{*}$ and $\Omega_{b c}^{\prime}, \Omega_{b c}^{*}$ states). The bag model results for the ground state baryon masses calculated in our work are laid out somewhat above the estimates [22] obtained in the nonrelativistic potential model based on the quark-diquark approximation. Relativistic approach [24] gives similar mass spectrum as ours but shifted approximately $70 \mathrm{MeV}$ upwards. The predictions based on the sum rules [26] are higher than our estimates by approximately $170 \mathrm{MeV}$ and $110 \mathrm{MeV}$ for the $\Xi_{b c}$ and $\Omega_{b c}$ families respectively. The difference between the baryon spectrum obtained in the paper [9] and the others is of qualitative character. It could look strange, but it is the direct consequence of the attempts to incorporate the mixing effects for the ground state baryons in the quark-diquark approximation to the bag model. We already know that in the ordinary approach the wave function mixing can play an important role in the calculations of the baryon mass spectra. However, in general, we cannot draw a direct link between the quark ordering in the spin coupling scheme and the corresponding diquark structure. Nevertheless, some correspondence between the two pictures is expected. For example, in the quark-diquark approximation to the potential model the physical $\Xi_{b c}^{\prime}$ and $\Omega_{b c}^{\prime}$ states are those with scalar $c b$ diquark [24], as could be expected from the analogy with the ordinary approach (see the 4th and 7th columns of Table 3). In the usual approach the interaction of the system consisting of two quarks with the third one is provided by the interaction of its individual constituents. The mixing of the wave functions is possible only when the interaction between the first and the third quarks is of different strength as compared with the interaction between the second and the third (as the quark becomes heavier its hyperfine interaction with other quarks decreases). When the mixing is present the correct mass splitting is achieved only after the diagonalization of the energy matrix. On the other hand, in the quark-diquark approximation some information about the initial structure of the diquark is lost, and, as a rule, the mixing of the ground state functions is absent [23]. Maybe some remnant of the mixing interaction of the ground states could exist, but practically it seems to be unnecessary. Since the baryon masses predicted in the paper [9] differ radically from the predictions obtained in the bag model with the state mixing effects taken into account (this work) and from the results obtained in the quark-diquark approximations to the potential model, it seems that the mixing effects in the work [9] have been heavily overestimated. Of course, in the calculation of energies of the excited baryons one is confronted with the mixing of various states, and in consistent calculations $[22,24]$ these mixing effects are taken into account.

As regards the results obtained in our work, first of all, we conclude that, as expected, the bag model shares many features of ordinary quark model. The main aim of this paper was to examine the heavy baryon ground state wave function mixing due to the colour-magnetic interaction in the framework of the modified bag model. We have found that the main features of the mixing interaction in the bag model are the same as in the ordinary nonrelativistic quark model. So, we can conclude that fully relativistic treatment of the light quarks in the bag has only minor influence on the state mixing properties. For the baryons consisting of three quarks of different flavour we cannot in general ignore the wave function mixing induced by the hyperfine colourmagnetic interaction. It can even cause sizable changes of the calculated hadronic properties. On the other hand, the widely accepted optimal basis can be built up by simply choosing the heaviest quark as the third one in the corresponding spin coupling scheme. The matrix of the interaction energy in this basis is approximately diagonal, and therefore the mixing effects in the baryon mass (energy) calculations can be neglected. If for any reason other than optimal basis is used, even in the baryon mass calculations the mixing effects must be taken into account.

\section{References}

[1] A. Chodos, R.L. Jaffe, K. Johnson, C.B. Thorn, and V.F. Weisskopf, Phys. Rev. D 9, 3471 (1974).

[2] A. Chodos, R.L. Jaffe, K. Johnson, and C.B. Thorn, Phys. Rev. D 10, 2599 (1974).

[3] T. DeGrand, R.L. Jaffe, K. Johnson, and J. Kiskis, Phys. Rev. D 12, 2060 (1975).

[4] P. Hasenfrantz and J. Kuti, Phys. Rep. 40, 75 (1978).

[5] C.E. DeTar and J.F. Donoghue, Ann. Rev. Nucl. Part. Sci. 33, 238 (1983).

[6] A.W. Thomas, Adv. Nucl. Phys. 13, 1 (1984).

[7] E.V. Shuryak, Phys. Lett. B 93, 134 (1980).

[8] D. Izatt, C. DeTar, and M. Stephenson, Nucl. Phys. B 199, 269 (1982).

[9] D. He, K. Qian, Y. Ding, X. Li, and P. Shen, Phys. Rev. D 70, 094004 (2004).

[10] J.F. Donoghue and K. Johnson, Phys. Rev. D 21, 1975 (1980).

[11] A. Bernotas and V. Šimonis, Nucl. Phys. A. 741, 179 (2004).

[12] T. Ito and Y. Matsui, Prog. Theor. Phys. 96, 659 (1996). 
[13] D. Sakharov, Pis'ma Zh. Eksp. Teor. Fiz. 21, 554 (1975) [JETP Lett. 21, 9 (1975)].

[14] J. Franklin, D.B. Lichtenberg, W. Namgung, and D. Carydas, Phys. Rev. D 24, 2910 (1981).

[15] N. Isgur, Phys. Rev. D 21, 779 (1980).

[16] J. Franklin, Phys. Rev. D 55, 425 (1997).

[17] F.E. Close, An Introduction to Quarks and Partons (Academic Press, 1979).

[18] A.P. Yutsis, I.B. Levinson, and V.V. Vanagas, The Theory of Angular Momentum (Israel Program for Scientific Translations, Jerusalem, 1962).

[19] L.C.L. Hollenberg and B.H.J. McKellar, J. Phys. G 16, 31 (1990).

[20] B. Aubert et al. (BABAR Collaboration), Phys. Rev. D 72, 052006 (2005).
[21] D. Acosta et al. (CDF Collaboration), Phys. Rev. Lett. 96, 202001 (2006).

[22] V.V. Kiselev, A.K. Likhoded, O.N. Pakhomova, and V.A. Saleev, Phys. Rev. D 66, 034030 (2002).

[23] D. Ebert, R.N. Faustov, and V.O. Galkin, Phys. Rev. D 72, 034026 (2005).

[24] D. Ebert, R.N. Faustov, V.O. Galkin, and A.P. Martynenko, Phys. Rev. D 66, 014008 (2002).

[25] C. Albertus, J.E. Amaro, E. Hernandez, and J. Nieves, Nucl. Phys. A 755, 439 (2005).

[26] D.B. Lichtenberg, R. Roncaglia, and E. Predazzi, Phys. Rev. D 53, 6678 (1996).

[27] R. Roncaglia, D.B. Lichtenberg, and E. Predazzi, Phys. Rev. D 52, 1722 (1995).

[28] Particle Data Group (W.-M. Yao et al.), J. Phys. G 33, 1 (2006).

\title{
SUNKIŲJŲ BARIONŲ MAIŠYMASIS MAIŠŲ (BAG) MODELIO SKAIČIAVIMUOSE
}

\author{
A. Bernotas, V. Šimonis \\ VU Teorines fizikos ir astronomijos institutas, Vilnius, Lietuva
}

\section{Santrauka}

Barionų, sudarytų iš trijų skirtingų aromatų kvarkų, pagrindinių būsenų papildomai klasifikacijai gali būti naudojami tarpiniai kvarkų poros sukiniai, tačiau dèl sukinio-sukinio tipo stipriosios sąveikos šie tarpiniai sukininiai momentai ne visada yra „geri“ kvantiniai skaičiai. „Fizikinès“ tokių dalelių (pavyzdžiui, $\Xi_{c}$ ir $\Xi_{c}^{\prime}$ ) banginès funkcijos bendru atveju yra pradinių ,matematinių“ funkcijų, charakterizuojamų tarpiniais sukiniais, superpozicija. Skaičiuojant tokių dalelių energiją, reikia atsižvelgti ir i nediagonaliụjų matricinių elementu ittaką. Yra žinoma, kad kai kuriais atvejais, sudarant barionu bangines funkcijas nereliatyvistiniame, potencialiniame modelyje, galima taip parinkti kvarkų sukinių surišimo schemą (pradžioje surišant lengvesnių kvarkų sukinius, o po to prie ju pridedant trečiojo, paties sunkiausio kvarko sukini), kad tarpiniai sukiniai išliktu santykinai ,gerais“ kvantiniais skaičiais. Siekiant išsiaiškinti, ar ir MIT maišų modelyje galioja panašūs dèsningumai, buvo atlikti nuodugnūs skaičiavimai, parenkant visas įmanomas kvarkų sukinių surišimo schemas ir atsižvelgiant i nediagonalius matricinius elementus. Paaiškejjo, kad čia taip pat egzistuoja optimali sukinių surišimo banginejje funkcijoje schema (tokia pat kaip ir potencialiniame modelyje), o kitose schemose pastebimas stiprus pradinių banginių funkcijų susimaišymas. Neblogas turimų eksperimentiniu duomenu sutapimas su modelio rezultatais rodo, kad maišų modelis, įskaičius jame hipersmulkiają spalvinę magnetinę sąveiką, gali būti naudojamas barionų nežinomų masių îverčiams. 